\title{
73. THEORETICAL 21-cm LINE PROFILES: COMPARISON WITH OBSERVATIONS
}

\author{
C. YUAN* \\ Massachusetts Institute of Technology, Cambridge, Mass., U.S.A.
}

\begin{abstract}
In order to make a direct comparison with observations of the 21-cm line of neutral hydrogen, theoretical profiles based on the ideas of the density-wave theory are constructed for a modified Schmidt model of the Galaxy and its theoretical spiral pattern. The comparison has covered galactic longitudes $l^{\mathrm{II}}=30^{\circ}-330^{\circ}$ with $10^{\circ}$ intervals in the galactic plane. Good agreement is found in most of the above directions.
\end{abstract}

\section{Introduction}

In this report, we shall briefly discuss the comparison of theoretical $21-\mathrm{cm}$ line profiles against observational data. Detailed results of the present study, however, will be published elsewhere (Yuan, 1970). The theoretical profiles are based on a large-scale spiral pattern for the Milky Way System, derived from an application of the densitywave theory of Lin and Shu $(1964,1966)$. Good agreement has been found in most of the galactic longitudes under study, which range from $l^{\mathrm{II}}=30^{\circ}$ to $l^{\mathrm{II}}=330^{\circ}$ at $10^{\circ}$ intervals. (Only 10 directions are presented in this paper.) We have thus obtained a spiral pattern for the Milky Way System that is consistent with both observations and the density wave concept.

There are, however, several other important implications in this agreement. The theoretical spiral pattern, at least from the solar vicinity outwards, depends sensitively upon the basic galactic model, composed of two related items, the surface density and the rotation curve. The decision on a basic model for this part of our galaxy, therefore becomes a crucial part of the present study. Furthermore, the construction of the profiles demands, besides the knowledge of the spiral pattern, the detailed information of the interstellar gas, which includes (1) the abundance distribution of neutral hydrogen, (2) the density contrast between the gas in the spiral arm and in the interarm region, (3) the systematic motion of the gas due to the presence of the spiral pattern, and (4) the turbulent speed of gas clouds. Our present study also contains a suggestion on these properties of gas as a whole.

\section{Theoretical Profiles}

The procedure of constructing theoretical profiles is indeed a reverse of the usual method of deducing spiral structure from the $21-\mathrm{cm}$ line observations. In the present study, the construction of the profiles is performed under the following three basic assumptions: (1) The spin temperature of neutral hydrogen is constant throughout the galaxy. (2) The velocity distribution of the gas is Gaussian at each point along

* Present address: City College of New York, N.Y., U.S.A. 
the line of sight. (3) The systematic motion and the spiral component of the volume density of the gas have a sinusoidal variation across the spiral arms, as specified by the linear theory of gas dynamics.

The first two assumptions immediately imply the following basic relation

$$
T_{b}\left(v, l^{\mathrm{II}}\right) \sim \int_{0}^{\infty} \varrho\left(r, l^{\mathrm{II}}\right) \exp \left\{-\frac{\left[v-V\left(r, l^{\mathrm{II}}\right)\right]^{2}}{2 c_{t}^{2}}\right\} \mathrm{d} r
$$

in which $T_{\mathrm{b}}$ is the brightness temperature, $v$ is the line-of-sight velocity and $r$ is the distance measured from the sun. The third assumption specifies the spiral component in the volume density $\rho$ and the systematic deviation of the velocity $V$ along the line of sight from that given by the circular motion in the basic model. Theoretical profiles are obtained by integrating the above equations for various values of $v$, with the turbulent speed $c_{t}$ and the spiral pattern also assigned. We shall proceed to describe these necessary quantities in the following sections.

\section{Theoretical Spiral Pattern}

Before we can calculate a theoretical spiral pattern, we must have a basic galactic model with reasonable accuracy. We shall adopt essentially the Schmidt model of 1965 , but with slight modifications from the solar vicinity outwards.

\section{A. BASIC DISK MODEL}

It is well known that the part of the rotation curve beyond the location of the sun could not be accurately obtained from observations. All the existing models have simply extended the rotation curve beyond that point by extrapolation. Such an extrapolation in galactic rotation may cause serious errors in the determination of the mass distribution. The density-wave theory, however, provides an additional check on this extrapolation. On the one hand, the arm spacing according to the theory is linearly related to the surface density. On the other hand, it can be obtained from the $21-\mathrm{cm}$ line profiles with the rotation curve. Thus, the extrapolation has to be so chosen that the corresponding surface density will reproduce an observed arm spacing. We shall apply this idea to the decision on the basic disk model.

First, we notice that the extrapolation adopted in the 1965 Schmidt model is somewhat unsatisfactory in this sense. With all the reasonable pattern speeds, the arm spacing beyond the location of the sun appears always too wide in comparison with observations (Yuan, 1969). A reduction in the surface density, and hence the rotation velocity, seems to be necessary beyond the sun. This may be accomplished by a mass cutoff to the Schmidt model.

We shall only summarize the final results here. With a cutoff at $19 \mathrm{kpc}$, the adopted model only deviates slightly from the 1965 Schmidt model. The Oort constants are only changed by less than $1 \%$. Local surface density is reduced by $15 \%$ and the circular velocity at $15 \mathrm{kpc}$ from the center is lower by $7 \%$. 


\section{B. SPIRAL PATTERN}

The theoretical spiral pattern is computed at the pattern speed $11.5 \mathrm{~km} \mathrm{~s}^{-1} \mathrm{kpc}^{-1}$. Inside the $10-\mathrm{kpc}$, this spiral pattern has no essential difference from the one published by Lin et al. (1969), but it winds up more tightly beyond that circle (solid curve, Figure 1). In order to account for the existence of the oval or other possible largescale distortions, some minor adjustment has been made to this theoretical pattern during the calculations of the profiles. The displacements of the arms radially from the original pattern are usually around $300 \mathrm{pc}$ but amount to about $500 \mathrm{pc}$ at a few places (dashed curve, Figure 1).

\section{The Adopted Model for the Gas Component}

\section{A. ABUNDANCE DISTRIBUTION}

The abundance distribution adopted in the calculations has no appreciable difference from the familiar diagram of Westerhout (Oort, 1965). The distribution as indicated in Figure 2 is characterized by its double-ring structure. The major ring is located at about $12 \mathrm{kpc}$, and can be easily recognized as those observed in the external galaxies (Roberts, 1967). The secondary ring is located at about $6 \mathrm{kpc}$. As we shall see next, this distribution is quite satisfactory as far as the large-scale structure of the gas is concerned.

\section{B. DENSITY CONTRAST AND SYSTEMATIC MOTION}

The linear theory of the response in gas provides a useful proportionality among the perturbation of gas density and the amplitudes of the tangential and the radial systematic motion (Lin et al., 1969). Once one quantity is known, the others follow immediately from the proportionality. The tangential systematic motion for the region inside the location of the sun can be directly obtained from the observed rotation curve, whereas the density contrast is relatively easy to estimate for the outer part of the Galaxy, from the observed profiles. Using these values as a guide, we have adopted the following values: The amplitudes of both components of the systematic motion are taken to be $7-9 \mathrm{~km} \mathrm{~s}^{-1}$. The density contrast varies from $2: 1$ at $5 \mathrm{kpc}$ to $5: 1$ at $15 \mathrm{kpc}$.

\section{TURBULENT SPEED OF GAS CLOUDS}

Turbulent speed is relatively insensitive in our calculation. A simple argument shows that the turbulent speed should roughly vary with the square root of the surface density. In the present study, the local turbulent speed is taken to be $8 \mathrm{~km} \mathrm{~s}^{-1}$. The value is increased to $13 \mathrm{~km} \mathrm{~s}^{-1}$ at $5 \mathrm{kpc}$ and reduced to $6 \mathrm{~km} \mathrm{~s}^{-1}$ at $15 \mathrm{kpc}$ from the center.

\section{ORION SPUR AND CARINA BRANCH}

In addition to the above-mentioned regular features, we have also included the Orion 


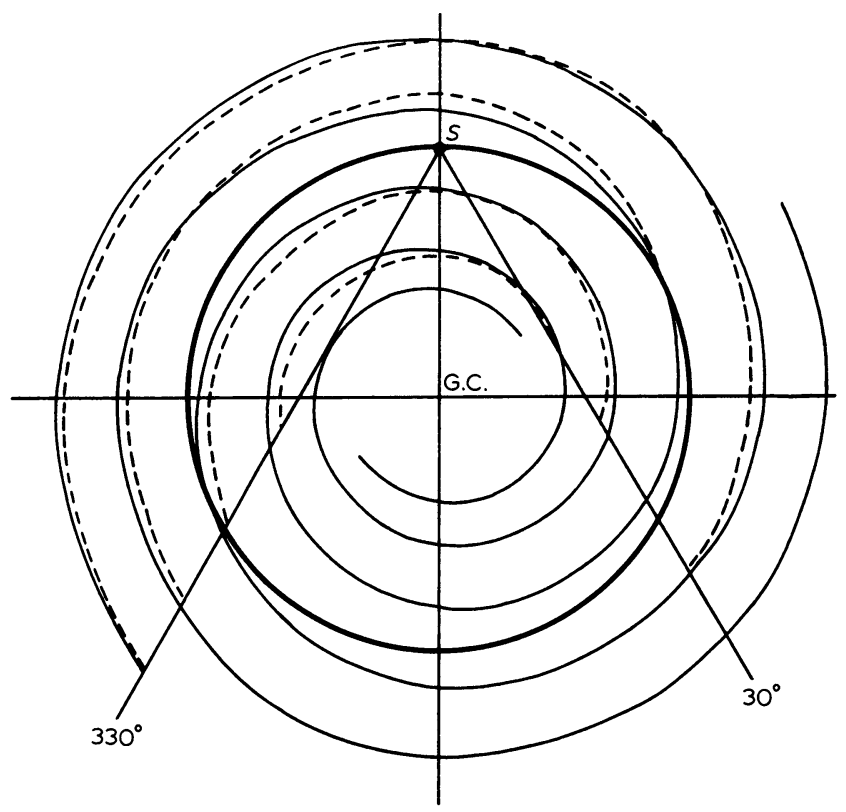

Fig. 1. Theoretical spiral pattern. The solid curve is the spiral pattern calculated at pattern speed $11.5 \mathrm{~km} \mathrm{~s}^{-1} \mathrm{kpc}^{-1}$ and based on the modified Schmidt model. The dashed curve is the spiral pattern actually used in constructing theoretical profiles.

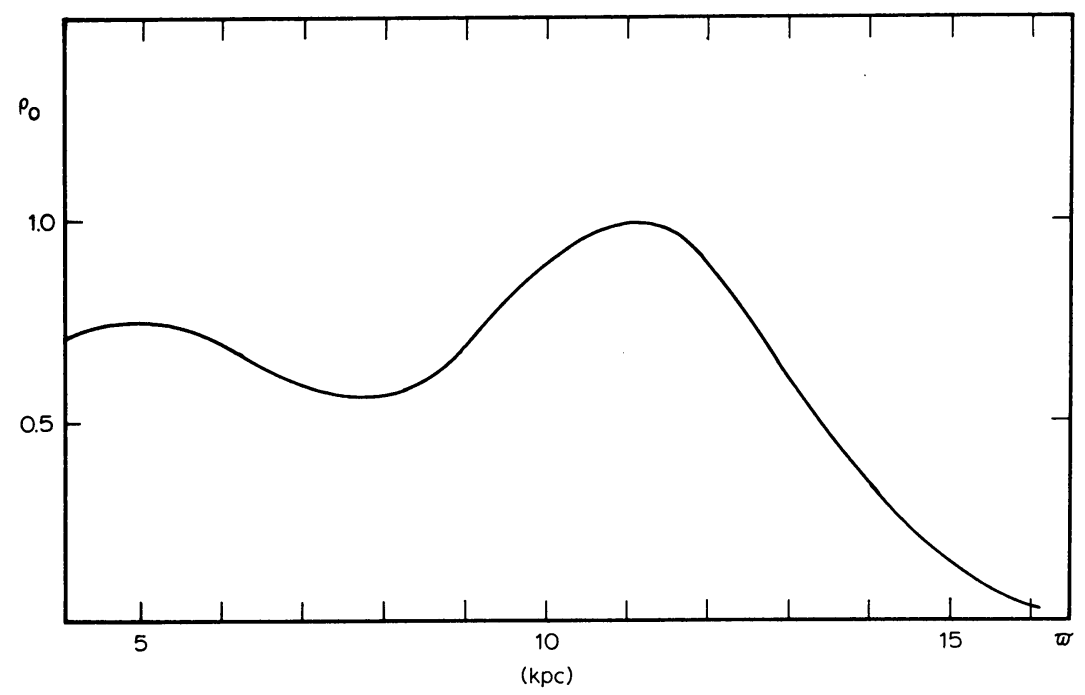

Fig. 2. The abundance distribution of the gas in the galactic plane. 


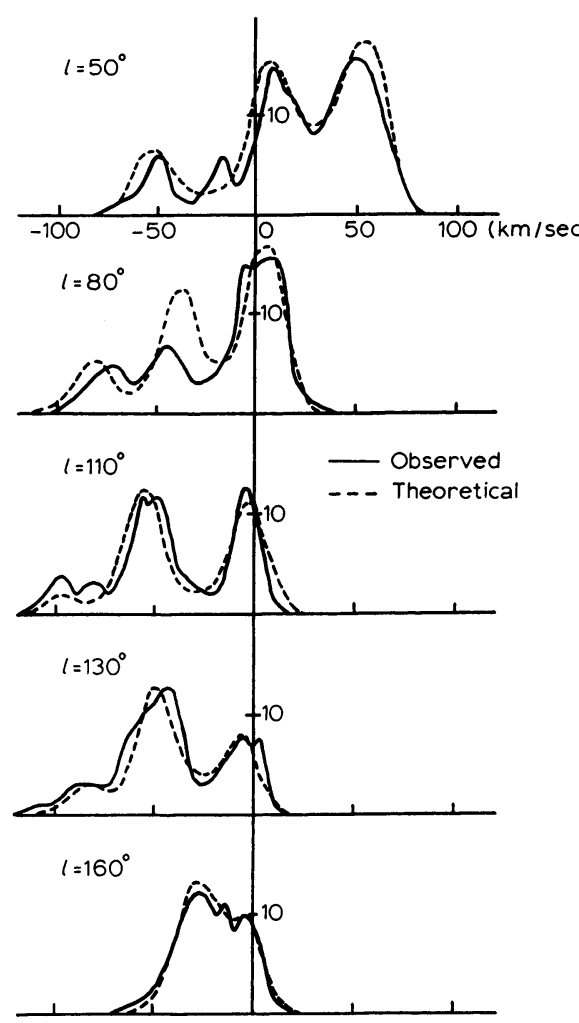

Fig. 3. Comparison with observations in the northern sky.

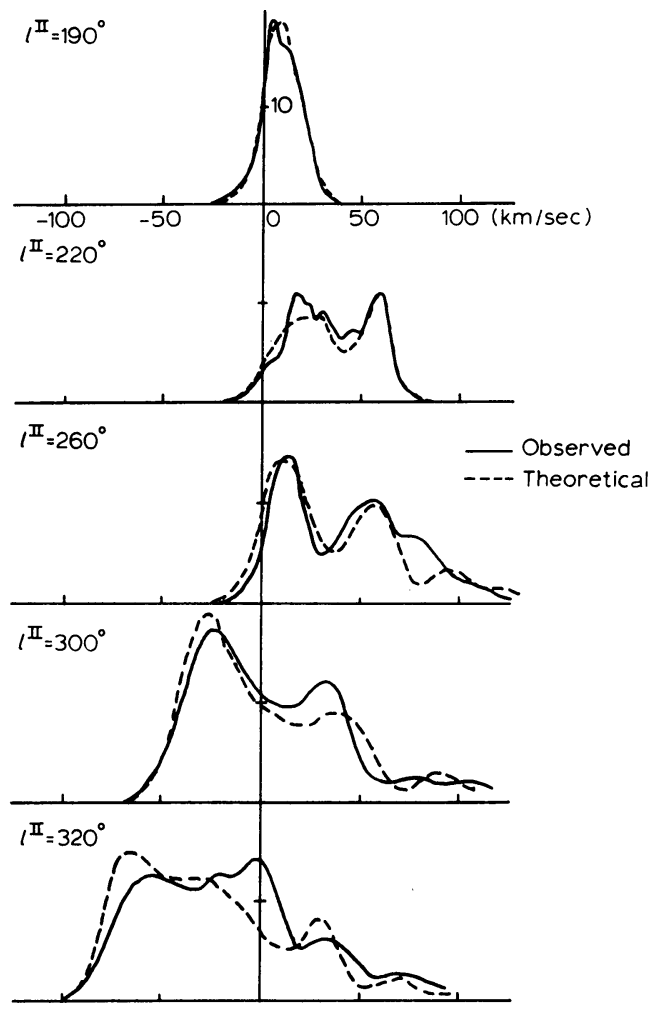

Fig. 4. Comparison with observations in the southern sky.

Spur in our calculation in order to see whether the 'local circular arm' in the northern sky can be reproduced by putting the gas along the optical Orion Spur. The answer is affirmative. In the same manner, we find a minor local feature of the Orion Spur in the southern sky. The gaseous branch in the Carina direction is also included in the study, for the purpose of comparison with Bok's extensive study in that region (1969). We have not found any evidence that the 'Carina branch' has extended into the 10-kpc circle to link itself to the Sagittarius Arm or the local Orion Spur.

\section{Results}

Detailed results in comparison will be published elsewhere (Yuan, 1969). In this paper, only ten directions, uniformly covering the galactic plane from $l^{\mathrm{II}}=30^{\circ}$ to $l^{\mathrm{II}}=330^{\circ}$, are presented (Figures 3 and 4). The locations and the intensities of the peaks and valleys are generally in good agreement with observations. This has greatly encouraged our theoretical investigation and also given us more confidence in the adopted large$\mathrm{s}$ cale spiral structure, determined with the guidance of the density-wave theory. 
Irregularities such as an interarm branch or spur occur quite commonly on the outer part of the galaxies. In those directions of irregularities, the agreement becomes poor. The Cygnus branch in the northern sky and the Carina branch in the southern sky are these examples. The existence of these branches in the outer part of the Galaxy indicates that other modes, possibly with multiple arms, may also be present in our galaxy.

\section{Acknowledgements}

The observational data in this paper are adopted from (1) Westerhout's Greenbank Survey for $l^{\mathrm{II}}=30^{\circ}-235^{\circ}$ (1965), (2) Hindman's data for $l^{\mathrm{II}}=235^{\circ}-300^{\circ}$ (1969), and (3) Kerr's data for $l^{I I}=300^{\circ}-330^{\circ}$ (1969). I would like to take this opportunity to thank Professor F. Kerr for making these southern data available to me before publication. I also wish to thank Professor C. C. Lin for his constant encouragement during this investigation and many most helpful discussions.

\section{References}

Bok, B.: 1969, private communication.

Kerr, F. J.: 1969, Australian J. Phys. Astrophys. Suppl., No. 9.

Lin, C. C. and Shu, F. H.: 1964, Astrophys. J. 140, 646.

Lin, C. C. and Shu, F. H.: 1966, Proc. Nat. Acad. Sci. U.S.A. 55, 229.

Lin, C. C., Shu, F. H., and Yuan, C.: 1969, Astrophys. J. 155, 721.

Oort, J. H.: 1965, Trans. IAU 12A, 789.

Roberts, M. S.: 1967, IAU Symposium No. 31, p. 189.

Schmidt, M.: 1965, Stars and Stellar Systems 5, 513.

Westerhout, G.: 1966, Maryland-Greenbank Galactic 21-cm Line Survey, 1st ed., University of Maryland.

Yuan, C.: 1969, Astrophys. J. 158, 871.

Yuan, C.: 1970, in preparation. 